\title{
TSH Measurement and Its Implications for Personalised Clinical Decision-Making
}

\author{
Rudolf Hoermann ${ }^{1}$ and John E. M. Midgley ${ }^{2}$ \\ ${ }^{1}$ Department of Nuclear Medicine, Klinikum Luedenscheid, Paulmannshoeherstraße 14, 58515 Luedenscheid, Germany \\ ${ }^{2}$ Consultancy Division, North Lakes Clinical, 6 High Wheatley, Ilkley, West Yorkshire LS29 8RX, UK
}

Correspondence should be addressed to Rudolf Hoermann, rudolf.hoermann@gmail.com

Received 5 October 2012; Accepted 15 November 2012

Academic Editor: Johannes W. Dietrich

Copyright ( 2012 R. Hoermann and J. E. M. Midgley. This is an open access article distributed under the Creative Commons Attribution License, which permits unrestricted use, distribution, and reproduction in any medium, provided the original work is properly cited.

\begin{abstract}
Advances in assay technology have promoted thyrotropin (TSH) measurements from participation in a multi-analyte assessment of thyroid function to a statistically defined screening parameter in its own right. While this approach has been successful in many ways, it has some grave limitations. This includes the basic question of what constitutes an agreed reference range and the fact that the population-based reference range by far exceeds the variation of the intraindividual set point. Both problems result in a potential misdiagnosis of normal and pathological thyroid function in a substantial proportion of patients. From a physiological perspective, TSH plays an integrated role in thyroid homeostasis. Few attempts have been made to adopt physiological insights into thyroid homeostasis for medical decision-making. Some emerging novel findings question the widely assumed log-linear TSH-FT 4 relationship over the entire thyroid function spectrum. This data favours more complex hierarchically structured models. With a better understanding of its role in thyroid homeostasis in thyroid health and disease, TSH can be revisited in the context of thyroid regulation. This, in turn, could help overcome some of the limitations arising from its isolated statistical use and offer new prospects towards a more personalised interpretation of thyroid test results.
\end{abstract}

\section{Background}

Recognition of the existence of the entity later described as thyroid-stimulating hormone (TSH) was first evident in the late 19th century [1]. In the late 1960s and early 70 s, academic immunoassays were developed and though initially cumbersome in use and somewhat insensitive, were quickly adopted as a diagnostic tool chiefly for determination of the hypothyroid state. From the mid 1980s to 1990s, steady developments in both convenience and sensitivity have so refined TSH assays that the concentrations of the hormone in subjects with normal thyroid function or with thyroid diseases are now readily quantifiable [2]. With the introduction of the third generation of commercial TSH assays into clinical routine diagnosis, assay sensitivity increased to $0.01 \mathrm{mU} / \mathrm{L}$, allowing a clear-cut discrimination of the normal thyroid state from both hypothyroidism and hyperthyroidism [3].

In the present paper, we address the current use of TSH as the dominant parameter in thyroid function testing, explain some major limitations of this approach, and attempt to suggest areas of possible improvement. The fact that TSH is a measure of regulatory control with widely varying setpoints among individuals rather than an outcome variable with a tight uniform range has various consequences for the statistical interpretation of this parameter, which differs sharply from other laboratory values. This, in turn, has further diagnostic, therapeutic, and prognostic implications that will be discussed. Improvements seem possible by respecting the regulatory individuality that is inherent in the parameter, as opposed to a population-based statistical use, and by advancing an understanding of the regulatory process and the interrelationship of TSH with circulating free thyroid hormones.

\section{Current Diagnostic Strategy}

Based on the methodological advances in TSH determination, the parameter has progressively evolved from its early adoption as an adjunct to the measurement of thyroid 
hormones to an exclusive parameter in its own right and consequently now dominates thyroid function testing. Modern diagnostic strategies have accordingly become heavily reliant on TSH measurement [2]. They have attributed various roles to TSH measurement as a screening tool, a therapeutic target in thyroid hormone treatment, and a prognostic marker [512]. With current disease classification based on TSH, this has introduced the subclinical states of hyperthyroidism or hypothyroidism that are defined by an abnormal TSH value in the presence of $\mathrm{FT}_{4}$ and $\mathrm{FT}_{3}$ values that still lay within their respective reference limits.

While this approach has been successful in many ways, it has also shifted the focus of TSH from its reactive and interactive role with thyroid hormones to an exclusive statistical parameter whose value is assumed to define the functional state of the subject. The present paper attempts to address some of the consequences of this paradigm shift and to assess some future perspectives for clinical decisionmaking.

\section{Use and Limitations of TSH as a Statistical Parameter}

While most problems of TSH measurement have been successfully resolved from the point of view of assay development and the analytical goals have been well defined, important issues that relate to the clinical application of the method still remain unsettled [13]. The assay performance does not resolve the problem that the immunological activity of TSH determined by the methods may not equate fully with its bioactivity [14]. This is important to note, because the bioactivity of TSH is subject to some variation depending on the level of thyroid function. This is a result of changes in carbohydrate content of the TSH molecule that is effected by the shift in function from hypothyroidism to hyperthyroidism [15]. Clinically, however, a slight dissociation between immunological and biological activity appears of minor significance, and there are currently no alternatives available to the immunoassays for routine clinical purpose.

This is less of a problem than the very basic question of what constitutes an agreed reference range. There has been a broad debate on the issue, particularly the setting of the upper reference limit, in which some authors argue for a wider reference range of approximately 0.3 to $4 \mathrm{mU} / \mathrm{L}$ and others advocate a more narrow interval with an upper limit of $2 \mathrm{mU} / \mathrm{L}$ [16-18]. The current state of affairs has recently been reviewed by Laurberg et al. [19]. It has also been pointed out that normal ranges and reference ranges are not necessarily to be considered as equivalent [20]. Importantly, TSH values are not normally distributed in a population, rather displaying a pattern of logarithmic normalisation and a skewed distribution. The latter findings have been questioned by arguing that if subjects with subtle thyroid disorders are included in the reference population, this may have distorted the observed normal distribution [21]. However, even when disease-free reference collectives were used, the disagreements still remain [22, 23]. Solely for illustration of the problem, but not for the support of assay validation per se, we have depicted the distribution of $\log$ TSH values that were obtained in a sample of euthyroid subjects (Figure 1(a)). The log transformation is generally required, because TSH values are not normally distributed (Figure 1(b)). For comparison, the normal distribution that was randomly generated from the mean and standard deviation of the sample is also shown (Figure 1(a)). The issue arises from the apparent distortion observed at the upper region of the TSH spectrum that has been frequently described, but not convincingly explained [21-23]. If one were to attribute the discrepancy to diseases such as unrecognized thyroid autoimmunity, a case could be made to adjust the spread of the normal distribution and consequently lower the upper reference limit. This is, however, not agreed nor is the reference interval [16-25].

The seemingly simple question of the disputed reference interval has far-reaching ramifications, which have been shown for instance in a study by Völzke et al. [24]. Employing a reference range established in their own investigation, according to the manufacturer's recommendation or as suggested by a US study [25], the authors reported a dramatic change in disease prevalence, from $7.7 \%$ to $24 \%$ in the case of diagnosed hypothyroidism and $0.8 \%$ to $3 \%$ for hyperthyroidism. This illustrates the grave consequences of the disagreement about TSH reference intervals that may result in clinical misclassification of a large proportion of patients.

\section{Possible Diagnostic Solutions}

A way out of the dilemma posited above could be envisaged by prospectively correlating TSH measurements to clinical outcome measures. There have indeed been a number of studies that relate a TSH screening value to various future events such as cardiovascular disease, particularly atrial fibrillation, osteoporosis, psychiatric disorders, and mortality [27-30]. However, while the statistical diagnostic approach may be appropriate on a large scale in a population study, it appears unsuitable for guiding clinical decisions in an individual patient. Andersen and colleagues found the thyroid hormone concentrations in normal subjects to vary much less in an individual than in the population [4]. Given the magnitude of this variation of up to $50 \%$, the distinction between a normal and pathological value cannot rely on the population-defined reference, but has to account for the individual patient's normal set point within the individual laboratory's reference range [4]. The high ratio of interindividual to intraindividual variability conflicts with any efforts to define a universally applicable clear cut-off and considerably limits the usefulness of the populationbased reference values for clinical decision-making. This problem can be graphically illustrated (Figure 2). The figure shows the distribution of $\log$ TSH in 150 euthyroid subjects that resembles the reference range in a population and, for comparison, the distribution that results when reducing the standard deviation by $50 \%$, which equals the spread of a normal intraindividual distribution according to Andersen et al. [4]. The movement of the narrow bell-shaped curve within the broader bell curve reflects the uncertainty about "true" normality that is inherent in the application of 


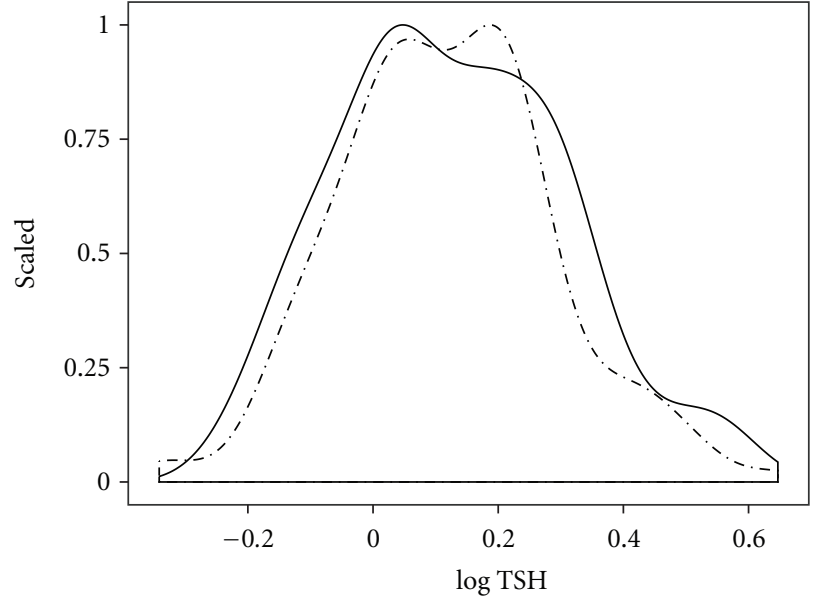

(a)

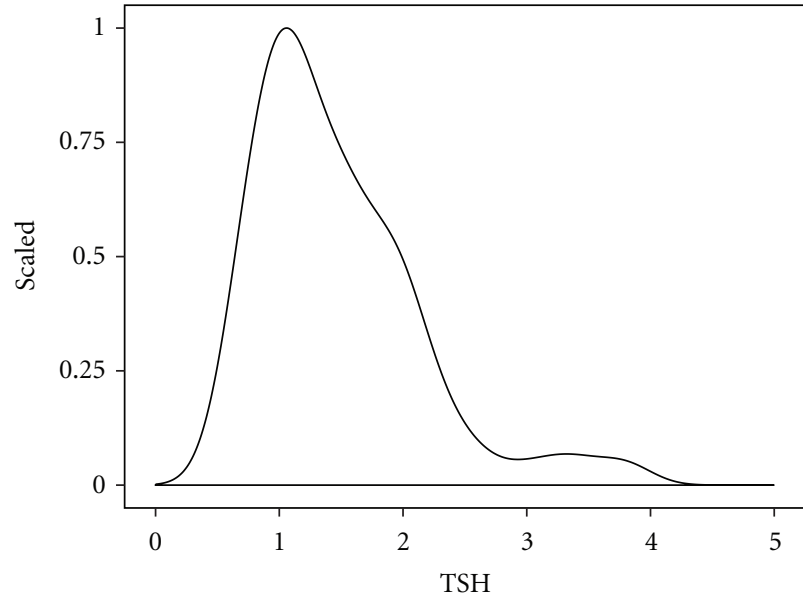

(b)

FIgURE 1: Distribution of $\log$ TSH (a) and TSH (b) in a clinical sample, compared to a random distribution. The distribution (solid line) was obtained from TSH measurements in 150 euthyroid subjects; a random distribution ((a), broken line) artificially generated by taking the mean and standard deviation of the sample.

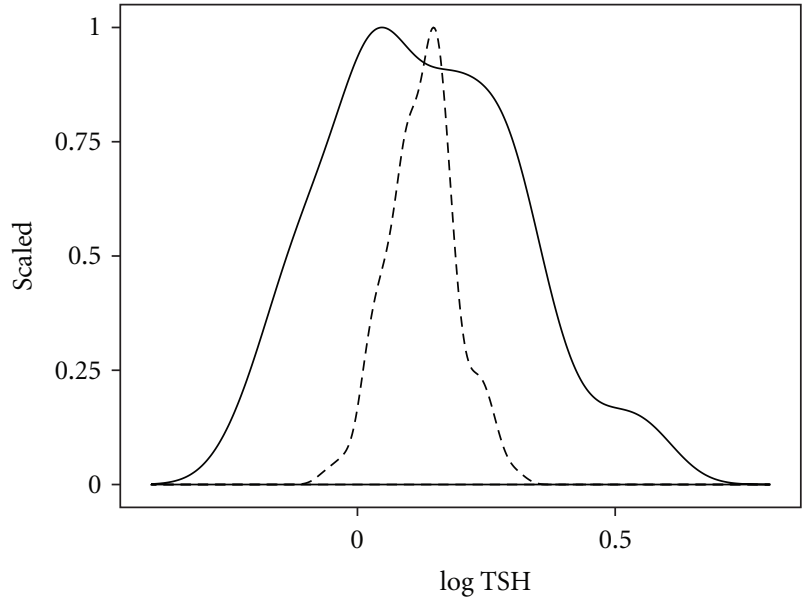

FIGURE 2: Distribution of $\log$ TSH in a group versus an individual. The TSH distribution in the group (solid line) was derived from 150 euthyroid subjects, with the individual spread (broken line) assumed to comprise $50 \%$ of the group variation (according to Andersen et al. [4]).

a population-based TSH reference system. This uncertainty on an individual basis exceeds by far that of many other laboratory parameters. Consequently, this makes the need for an individualised approach towards thyroid function testing more compelling than in other conditions of the subjects.

Another limiting factor is the significant fluctuation of the TSH levels over the course of time, which has been reported to occur in subclinical hypothyroidism [31]. The TSH value has, therefore, to change by at least $30 \%$ to discriminate between a natural variation and a real progression [31]. Additional influences such as gender, age, or time of sampling are less pronounced [32]. Also, in predictive long-term studies that have relied on a single initial TSH value this may not be an integer of the thyroid function status over the whole time period, but subject to change, as the dysfunction could have worsened or improved since the measurement was obtained [33]. Interestingly, monitoring intervals have been shown to directly influence the outcome [34]. Conditions that directly alter or interfere with the reliability of thyroid test results such as severe nonthyroid illness, pregnancy, severe renal insufficiency, and pituitary disorders are another topic [35].

The issue of thyroid hormone measurement in pregnancy has only recently been addressed by two updated guidelines issued by the American Thyroid Association in conjunction with the American Society of Clinical Endocrinologists and independently by the Endocrine Society following one year earlier pregnancy-specific guidelines [5, 36, 37]. Pregnancy exemplifies a choice between $\mathrm{FT}_{4}$ measurement and reliance on measuring total $\mathrm{T}_{4}\left(\mathrm{TT}_{4}\right)$, corrected for transport proteins, predominantly TBG. The new guidelines tend to favour $\mathrm{TT}_{4}$ measurement over $\mathrm{FT}_{4}$ methods, with one of them making this an explicit recommendation [5]. $\mathrm{FT}_{4}$ immunoassay measurement has historically been perceived as inaccurate in pregnant women by many authors $[5,36$, 37]. However, when scrutinized more closely, the assumption of a superiority of a $\mathrm{TT}_{4}$-TBG combination in pregnancy compared to $\mathrm{FT}_{4}$, despite acknowledged limitations in assay validation, does not hold true. In pregnancy, a combination of $\mathrm{TBG}$ and $\mathrm{TT}_{4}$ confers a variation to the reference range that is by far greater, compared to direct $\mathrm{FT}_{4}$ measurement, and therefore less accurate in defining the "normal" range in this situation in a patient. Spreads can be compared by the standardized variation (SD/Mean) or the ratio of the 97.5 percentile to the 2.5 percentile of the range [38]. In one study, when calculating the values on the basis of the generally accepted ranges for the parameters, the ratio was 3.0 in $\mathrm{TT}_{4}$ and only 2.4 in $\mathrm{FT}_{4}[38,39]$. The smaller spread 
for $\mathrm{FT}_{4}$ suggests that the $\mathrm{TT}_{4}$ reference range is compromised by uncorrected TBG variation [38]. Since TBG values are higher in pregnancy, the effect of their variation has a greater impact in this condition, but it is equally true for the nonpregnant situation. Hence, the choice here is between measurement of $\mathrm{TT}_{4}$, which is clearly diagnostically inferior, or $\mathrm{FT}_{4}$, which sometimes lacks careful validation among different manufacturers as a prerequisite to its adequate use. The general reliability of $\mathrm{FT}_{4}$ results obtained with the assays during pregnancy has, however, been verified by comparison with equilibrium dialysis ID-LC/tandem MS candidate reference measurement [40].

We would prefer an $\mathrm{FT}_{4}$ method applied with methodological rigor and careful attention to range setting and consider the novel recommendation to measure $\mathrm{TT}_{4}$ in pregnancy an ill-advised backward step, apart from practical considerations that most routine laboratory by now have wide experience with $\mathrm{FT}_{4}$ assays, but not $\mathrm{TT}_{4} . \mathrm{FT}_{4}$ should be used in conjunction with TSH, and interpretation in pregnancy must be based on gestation time-specific reference intervals of the two parameters and with recognition of variability in assay performance $[5,37,38,41]$. A lack of availability and clinical experience currently limit the use of novel mass-spectrometric (LC-MS/MS) techniques [37, 42]. Though inconsistency of assay performance is a recognised obstacle to optimal diagnostic protocols, a full discussion of assay validation is beyond the scope of this paper $[43,44]$.

Although the limitations of the current use of TSH measurement have been well documented and subject to a number of excellent reviews, we generally lack suggestions as to how to improve clinical management strategies. Obviously, a desirable aim would be to move from a populationbased statistical perspective to a more personalised approach. While, to an extent, this seems to be what we aim at in good clinical practise when considering the whole picture including laboratory tests, clinical examination, and subjective well-being of the patient, it does not appear to be adequately reflected in current evidence-based guidelines [5].

We conclude that advances in assay techniques have unduly promoted TSH measurement to its current role as an exclusive statistical estimate in its own right and the most important single parameter in thyroid function testing, thereby optimising both convenience and cost. However, the predominant use of TSH as a statistical parameter has some severe shortcomings that limit its clinical usefulness in a given patient. A revision may be needed to reconcile TSH measurement with the challenge of not only evidence-based but personalised medicine.

\section{Role of TSH as a Physiological Parameter}

From a physiological perspective, TSH is not an isolated player, but an integrated part in a complex system of thyroid homeostasis. Thyroid homeostasis is maintained by the negative feedback that is given by the peripheral concentrations of the hormones $\mathrm{FT}_{4}$ and $\mathrm{FT}_{3}$ to regulatory centres in the hypothalamus and pituitary gland that govern thyroid hormone production via the adjusted release of a thyroid-stimulating hormone (TSH) into the circulation.
A better understanding of thyroid hormone homeostasis including the role of TSH in the context may therefore aid in improving the diagnostic reliability of TSH measurement from both a methodological and clinical perspective. Modern thyroid function testing has the danger of exploiting TSH feedback out of context and has replaced the endpointbased definition (measuring $\mathrm{FT}_{4}$ and $\mathrm{FT}_{3}$ ) by a TSHcentred protocol, as discussed above. This paradigm shift has made interpretation of test results seemingly easy. From a homeostatic perspective, however, dysfunctional states, such as hypothyroidism or hyperthyroidism present adaptive challenges to the homeostatic system that result in a complex and concerted pattern of actions to restore euthyroidism or at least ameliorate the situation. This approach sees TSH as an integral part of the homeostatic system rather than a separate or even dominant determinant.

Attempts have been made to adopt physiological insights into thyroid homeostasis for medical decision-making. Some authors have developed fundamental mathematical models of thyroid homeostasis; others have exploited the interplay of $\mathrm{FT}_{3}, \mathrm{FT}_{4}$, and TSH to reduce the wide spread of exclusive TSH measurements [45-48]. Earlier studies have shown that $\mathrm{FT}_{4}$ has a relatively more pronounced influence on pituitary TSH secretion than does $\mathrm{FT}_{3}$ [49]. This is somewhat paradoxical, because $\mathrm{FT}_{3}$ is the more biologically active hormone of the two, and $\mathrm{T}_{4}$ only gains its activity following its deiodination, a process known as conversion and regulated by various deiodinases [49]. The phenomenon has been explained by the specific properties of pituitary deiodinase type 2 which allows efficient conversion to continue with high $\mathrm{FT}_{4}$ concentrations at the central levels, whereas a downregulation of the enzyme occurs in peripheral tissues [49]. The natural relationship between TSH and $\mathrm{FT}_{4}$ has been widely assumed to be both universal over the whole thyroid function spectrum and log linear [50-53]. A recent study has challenged this widely held view of a single all-encompassing log linear correlation between TSH and $\mathrm{FT}_{4}$ [26]. In this study, we demonstrated a more complex nonlinear interrelationship between the two parameters in a large clinical sample (Figure 3) [26]. While there has been some criticism as to the retrospective study design, heterogeneous study population, and reliability of $\mathrm{FT}_{4}$ measurement, the findings have recently been independently confirmed $[54,55]$. If the relationship is truly non-linear and noncontinuous, the system of thyroid hormone feedback control has to be reconsidered, because it is critical to the current role of TSH, for instance, when used as an exclusive therapeutic target establishing dose adequacy in patients treated with exogeneous $\mathrm{L}_{-} \mathrm{T}_{4}$.

\section{Implications for Regulation and Diagnostic Strategies}

Taken together, the studies described have several implications for our understanding of both hypothalamic pituitary thyroid regulation and the clinical interpretation of $\mathrm{TSH}$ results. Firstly, they suggest that a simple log linear correlation may not reliably represent the relationship between 
TSH and $\mathrm{FT}_{4}$. Hence, understanding of the interplay of TSH and $\mathrm{FT}_{4}$ requires more complex modelling. Secondly, they suggest that pituitary thyroid feedback control is not convincingly represented by an all-encompassing single process, but better characterised by a hierarchically structured type of control with distinct patterns of operative mechanisms that are unique to different functional states.

This assumption is further supported when reviewing the literature on the molecular aspects of thyroid hormone regulation. Earlier studies have shown the existence of isoforms of thyroid hormone receptors (TR) in different tissues [56]. TRß2 is exclusively expressed in the central nervous system including the pituitary and shows an up to 10-fold enhanced sensitivity to thyroid hormones [57-59]. Consequently, the pituitary is able to sensitively monitor small increases in $\mathrm{T}_{4}$ supply and to counteract $\mathrm{T}_{4}$ overproduction in advance of the occurrence of any effect on the peripheral tissues. Basically, TSH dampens its own action in anticipation of its effect on $\mathrm{T}_{4}$ secretion via an ultrashort feedback loop, thereby also giving rise to a pulsatile secretory pattern $[26,46$, $60]$. The activities and contributions of various deiodinases controlling conversion of $\mathrm{T}_{3}$ from $\mathrm{T}_{4}$ have also been reported to differ in the hypothyroid and hyperthyroid state [61]. TRH is key in orchestrating a response in the event of hormone undersupply. It stimulates pituitary TSH secretion and, additionally, modulates its bioactivity $[15,62]$. Studies with transgenic animals have demonstrated regulatory varying roles for both thyroid hormone feedback and TRH $[63,64]$. The distinct mechanisms unravelled by the molecular studies are difficult to reconcile with the assumption of a single allencompassing gradient of the relationship between TSH and $\mathrm{FT}_{4}$, but would naturally explain a complex and hierarchical pattern of the regulation. It also implies that correlating a given TSH value and $\mathrm{FT}_{4}$ level may not be a simple and straightforward process, as previously thought.

\section{Clinical Perspective of TSH Measurement towards a Personalised Approach}

The promotion of TSH into a parameter of its own right, with a statistical distribution and correlation to clinical outcomes and targets, has on the one hand greatly facilitated thyroid function testing. On the other hand, it has failed to consider adequately the individual set points of the pituitary thyroid axis that are far more narrowly defined than the broad interindividual distribution of the parameter (Figure 2). This failure may have consequences for clinical decision-making, in terms of not only misclassification of health and disease, as mentioned above, but also misjudging dose adequacy in thyroid hormone replacement. The latter is in particular need of being addressed because clinical studies in treated patients have invariably demonstrated a disturbingly high rate of patients that were dissatisfied with their mode of treatment $[65,66]$. Questions such as of $T_{4}$ monotherapy versus $T_{3} / T_{4}$ combination treatment, we believe, cannot be assessed on statistical grounds alone, without a solid understanding of the underlying regulatory process. The therapeutic requirements may differ in different conditions or populations, and a slightly elevated TSH that

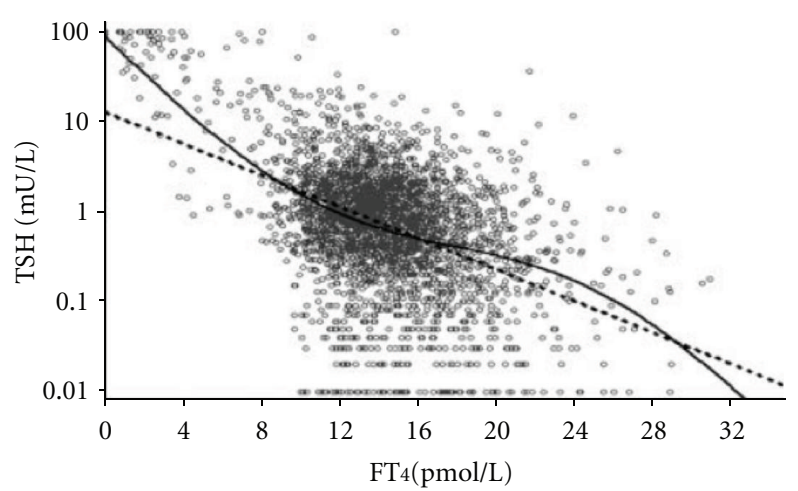

Figure 3: Comparison of a nonlinear model with the log linear standard model in a large clinical data set. The superior fit of the non-linear model was proven by comparative curve fitting, as shown in [26]. The figure is reprinted by permission of the publisher Bioscientia (http://www.eje-online.org/site/misc/permissions_commercial_reprints.xhtml).

is indicative of subclinical hypothyroidism per definition cannot be used as a universal marker, because it has been reported to increase mortality in some populations, whereas it promoted longevity in others, particularly in the elderly $[11,12,67,68]$.

A remedy may be to revisit the roots of TSH determination that has originated as a thyroid feedback regulator in thyroid homeostasis. While studies devoted exclusively to TSH abound in the literature, the interrelationship of thyroid parameters and their homeostatic roles have received scant attention [26, 46-55, 69-71]. Even when both TSH and thyroid hormone measurements were available on a large scale, as was for instance the case in a recent study on cardiovascular mortality, their use was limited to the purpose of defining subclinical states of thyroid dysfunction, but did not include a broader and more detailed analysis of the interplay of the parameters on the outcome [11, 72]. The latter approach seems more liable to advance our understanding and propel TSH measurement towards a more personalised approach of thyroid function testing. It does, however, require as a prerequisite a better understanding of thyroid hormone feedback regulation, which is currently lacking, but is evolving in new directions $[26,54,55]$. The new models allow for distinctly different adaptive homeostatic processes to be activated in either euthyroidism, hypothyroidism, or hyperthyroidism. Each state is thereby defined as a qualitatively different entity with a distinct pattern of homeostatically operative mechanisms and equilibria.

A dynamic TSH response that depends on the prevailing thyroid function state closely links the parameter to the circulating thyroid hormones, mainly $\mathrm{FT}_{4}$. Genetic variability may also play a role in shaping the relationship, for example, via deiodinase polymorphisms $[70,71]$.

An $\mathrm{FT}_{4}$-corrected TSH could, therefore, reduce the variation of test results. The complex nature of the $\mathrm{FT}_{4}$ TSH relationship requires more advanced algorithms to be established and clinically tested. A simplified tentative 


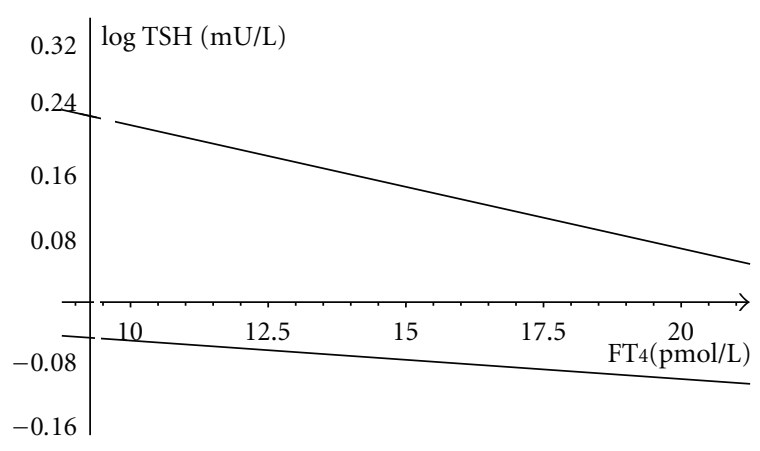

FIgUre 4: $\mathrm{FT}_{4}$ adjusted TSH band in the euthyroid range. The linear regression fitted to the euthyroid sample $(n=150)$ is described by the equation $( \pm \mathrm{SE}), \log \mathrm{TSH}=-0.014( \pm 0.006) * \mathrm{FT}_{4}+0.36$ $( \pm 0.1)$. The lines indicate the upper and lower $2 \mathrm{SD}$ range of the regression line.

representation of the idea is shown in Figure 4, based on a fitted log linear model to the euthyroid sample. Smaller pilot studies in clinical settings have indicated that a correlated calibration of TSH and $\mathrm{FT}_{4}$ or $\mathrm{FT}_{3}$ measurements outperforms the standard procedure in reducing the diagnostic spread [47, 48]. Jostel et al. [47] have demonstrated that an $\mathrm{FT}_{4}$-adjusted TSH, termed TSH index by the authors and calculated according to the formula TSHI $=\log \mathrm{TSH}+0.13 *$ $\mathrm{FT}_{4}$, facilitated the diagnosis of secondary hypothyroidism, providing an accurate estimate of the severity of TSH deficiency in hypopituitarism. The TSH index has not been extensively validated in primary thyroid disorders [47]. Meier et al. [48] introduced a bivariate zonal representation for the normal ranges of thyroid function tests. The authors validated the method in 257 volunteers and demonstrated an improved diagnostic accuracy, based on $\mathrm{TSH}_{-} \mathrm{FT}_{3}$ zones, in borderline cases, compared to the uncorrelated standard approach using the normal distributions of TSH and $\mathrm{FT}_{3}$ [48]. Complex cybernetic models could also tie $\mathrm{FT}_{4}$ and TSH together in a more accurate manner, to establish regulationbased discriminatory cut-offs of the parameters and increase their diagnostic value, but these have not been broadly tested either $[45,46]$.

In summary, correlative studies are few, compared to the vast literature on TSH. As a result, they have failed to gain broader acceptance and did not include more elaborate modelling.

The availability of presumably more reliable assays for $\mathrm{FT}_{4}$ based on LC-MS/MS may be advantageous for the development of such ideas [42, 53, 73]. However, evaluating $\mathrm{FT}_{4}$ assays and comparing different methods by conflating sera encompassing the whole spectrum of thyroid function, as has been proposed by some authors [53, 73], is not supported in our view, given that the three physiological areas have their own responses and any necessarily arbitrary mixture of subjects must have a distorting influence on the results. Despite some promising reports, there are no large trials currently available to reliably assess the clinical improvement introduced by the new methods, compared to existing technology.
In this setting, TSH screening could still have an important role as a first line test, in order to sensitively detect abnormalities, but the therapeutic decision should be made more specifically by interpreting values in the context of the underlying conditions and homeostatic equilibria. A slightly elevated TSH in subclinical hypothyroidism may accompany the successful adaptive response in some patients, but signal a failed adaption in others. Prognostic studies do not support an indiscriminate use of TSH as a therapeutic target $[12,67]$. Elevated TSH in subclinical hypothyroidism has been implicated to signal an adverse prognostic outcome in large population studies, but has also been documented as a marker of longevity in a very aged population [11, 12, 67]. This discrepancy argues against the adoption of a simplistic universal use of TSH values that are slightly outside the reference range, but are also taken out of context to the individual or particular situation. While interesting statistical associations have been documented between TSH and various clinical outcomes, a deeper understanding at the physiological and regulatory level is required to put them into context and improve clinical decision-making.

\section{Summary}

The current mainly statistically based use of TSH in thyroid function testing has some severe limitations, including the problem of nonexisting agreed reference limits, a lack of consideration of individual set points, and a prognostic heterogeneity in different populations. In the light of recent molecular and clinical evidence, revised and more refined modelling of the interrelations of TSH and thyroid hormones appears to be both a physiological requirement and a promising avenue towards an overdue reevaluation of TSH as an exclusive diagnostic standard and therapeutic target. Based on studies on the $\mathrm{FT}_{4}$-TSH relationship including our own work and a review of the molecular mechanisms described in the literature we propose a complex non-linear model and hierarchical structure of the thyroid hormone TSH interaction. We promote the interpretation of TSH results in close correlation to the thyroid hormone milieu to reduce the uncertainty of interpretation. While a few smaller studies could demonstrate a conceptually higher accuracy, compared to the standard diagnostic procedure, a possible broader application of such concepts awaits further methodological and clinical evaluation.

\section{Conflict of Interests}

The authors declare that there is no conflict of interests that could be perceived as prejudicing the impartiality of the research reported.

\section{References}

[1] S. Schwartzbach and G. Uhlenhuth, "Anterior lobe substance, the thyroid stimulator; effect of feeding anterior lobe upon amphibian metamorphosis," Experimental Biology and Medicine, vol. 26, no. 2, pp. 151-152, 1928. 
[2] L. M. Demers and C. A. Spencer, "Thyrotropin/Thyroid Stimulating Hormone (TSH) measurement," Thyroid, vol. 13, pp. 33-44, 2003.

[3] C. A. Spencer, D. Schwarzbein, R. B. Guttler, J. S. LoPresti, and J. T. Nicoloff, "Thyrotropin (TSH)-releasing hormone stimulation test responses employing third and fourth generation TSH assays," The Journal of Clinical Endocrinology and Metabolism, vol. 76, no. 2, pp. 494-498, 1993.

[4] S. Andersen, K. M. Pedersen, N. H. Bruun, and P. Laurberg, "Narrow individual variations in serum $\mathrm{T}_{4}$ and $\mathrm{T}_{3}$ in normal subjects: a clue to the understanding of subclinical thyroid disease," The Journal of Clinical Endocrinology and Metabolism, vol. 87, no. 3, pp. 1068-1072, 2002.

[5] J. R. Garber, R. H. Cobin, H. Gharib et al., "Clinical practice guidelines for hypothyroidism in adults co-sponsored by the American Association of Clinical Endocrinologists (AACE) and the American Thyroid Association, Inc. (ATA)," Thyroid, vol. 22, no. 12, pp. 1200-1235, 2012.

[6] P. R. Larsen, "Thyroid-pituitary interaction. Feedback regulation of thyrotropin secretion by thyroid hormones," The New England Journal of Medicine, vol. 306, no. 1, pp. 23-32, 1982.

[7] A. C. Waring, S. Harrison, M. H. Samuels et al., "Thyroid function and mortality in older men: a prospective study," The Journal of Clinical Endocrinology and Metabolism, vol. 97, no. 3, pp. 862-870, 2012.

[8] M. P. Rozing, J. J. Houwing-Duistermaat, P. E. Slagboom et al., "Familial longevity is associated with decreased thyroid function," The Journal of Clinical Endocrinology and Metabolism, vol. 95, no. 11, pp. 4979-4984, 2010.

[9] M. R. Haymart, D. J. Repplinger, G. E. Leverson et al., "Higher serum thyroid stimulating hormone level in thyroid nodule patients is associated with greater risks of differentiated thyroid cancer and advanced tumor stage," The Journal of Clinical Endocrinology and Metabolism, vol. 93, no. 3, pp. 809814, 2008.

[10] J. V. Parle, P. Maisonneuve, M. C. Sheppard, P. Boyle, and J. A. Franklyn, "Prediction of all-cause and cardiovascular mortality in elderly people from one low serum thyrotropin result: a 10-year cohort study," The Lancet, vol. 358, no. 9285, pp. 861-865, 2001.

[11] T. H. Collet, J. Gussekloo, D. C. Bauer et al., "Subclinical hyperthyroidism and the risk of coronary heart disease and mortality," Archives of Internal Medicine, vol. 172, no. 10, pp. 799-809, 2012.

[12] H. Völzke, C. Schwahn, H. Wallaschofski, and M. Dörr, "Review: the association of thyroid dysfunction with all-cause and circulatory mortality: is there a causal relationship?" The Journal of Clinical Endocrinology and Metabolism, vol. 92, no. 7, pp. 2421-2429, 2007.

[13] J. Karmisholt, S. Andersen, and P. Laurberg, "Analytical goals for thyroid function tests when monitoring patients with untreated subclinical hypothyroidism," Scandinavian Journal of Clinical and Laboratory Investigation, vol. 70, no. 4, pp. 264268, 2010.

[14] L. Persani, M. Terzolo, C. Asteria, F. Orlandi, A. Angeli, and P. Beck-Peccoz, "Circadian variations of thyrotropin bioactivity in normal subjects and patients with primary hypothyroidism," The Journal of Clinical Endocrinology and Metabolism, vol. 80, no. 9, pp. 2722-2728, 1995.

[15] L. Persani, S. Borgato, R. Romoli, C. Asteria, A. Pizzocaro, and P. Beck-Peccoz, "Changes in the degree of sialylation of carbohydrate chains modify the biological properties of circulating thyrotropin isoforms in various physiological and pathological states," The Journal of Clinical Endocrinology and Metabolism, vol. 83, no. 7, pp. 2486-2492, 1998.

[16] M. I. Surks, G. Goswami, and G. H. Daniels, "The thyrotropin reference range should remain unchanged," The Journal of Clinical Endocrinology and Metabolism, vol. 90, no. 9, pp. 5489-5496, 2005.

[17] G. Brabant, P. Beck-Peccoz, B. Jarzab et al., "Is there a need to redefine the upper normal limit of TSH?" European Journal of Endocrinology, vol. 154, no. 5, pp. 633-637, 2006.

[18] L. Wartofsky and R. A. Dickey, "The evidence for a narrower thyrotropin reference range is compelling," The Journal of Clinical Endocrinology and Metabolism, vol. 90, no. 9, pp. 5483-5488, 2005.

[19] P. Laurberg, S. Andersen, A. Carlé, J. Karmisholt, N. Knudsen, and I. B. Pedersen, "The TSH upper reference limit: where are we at?" Nature Reviews Endocrinology, vol. 7, no. 4, pp. 232239, 2011.

[20] R. A. Dickey, L. Wartofsky, and S. Feld, "Optimal thyrotropin level: normal ranges and reference intervals are not equivalent," Thyroid, vol. 15, no. 9, pp. 1035-1039, 2005.

[21] C. A. Spencer, J. G. Hollowell, M. Kazarosyan, and L. E. Braverman, "National Health and Nutrition Examination Survey III Thyroid-Stimulating Hormone (TSH)-thyroperoxidase antibody relationships demonstrate that TSH upper reference limits may be skewed by occult thyroid dysfunction," The Journal of Clinical Endocrinology and Metabolism, vol. 92, no. 11, pp. 4236-4240, 2007.

[22] M. I. Surks and J. G. Hollowell, "Age-specific distribution of serum thyrotropin and antithyroid antibodies in the U.S. population: implications for the prevalence of subclinical hypothyroidism," The Journal of Clinical Endocrinology and Metabolism, vol. 92, no. 12, pp. 4575-4582, 2007.

[23] T. E. Hamilton, S. Davis, L. Onstad, and K. J. Kopecky, “Thyrotropin levels in a population with no clinical, autoantibody, or ultrasonographic evidence of thyroid disease: implications for the diagnosis of subclinical hypothyroidism," The Journal of Clinical Endocrinology and Metabolism, vol. 93, no. 4, pp. 1224-1230, 2008.

[24] H. Völzke, D. Alte, T. Kohlmann et al., "Reference intervals of serum thyroid function tests in a previously iodine-deficient area," Thyroid, vol. 15, no. 3, pp. 279-285, 2005.

[25] J. G. Hollowell, N. W. Staehling, W. Dana Flanders et al., "Serum TSH, T4, and thyroid antibodies in the United States population (1988 to 1994): National Health and Nutrition Examination Survey (NHANES III)," The Journal of Clinical Endocrinology and Metabolism, vol. 87, no. 2, pp. 489-499, 2002.

[26] R. Hoermann, W. Eckl, C. Hoermann, and R. Larisch, "Complex relationship between free thyroxine and TSH in the regulation of thyroid function," European Journal of Endocrinology, vol. 162, no. 6, pp. 1123-1129, 2010.

[27] C. T. Sawin, A. Geller, P. A. Wolf et al., "Low serum thyrotropin concentrations as a risk factor for atrial fibrillation in older persons," The New England Journal of Medicine, vol. 331, no. 19, pp. 1249-1252, 1994.

[28] R. W. Flynn, S. R. Bonellie, R. T. Jung, T. M. MacDonald, A. D. Morris, and G. P. Leese, "Serum thyroid-stimulating hormone concentration and morbidity from cardiovascular disease and fractures in patients on long-term thyroxine therapy," The Journal of Clinical Endocrinology and Metabolism, vol. 95, no. 1, pp. 186-193, 2010.

[29] M. D. Williams, R. Harris, C. M. Dayan, J. Evans, J. Gallacher, and Y. Ben-Shlomo, "Thyroid function and the natural history of depression: findings from the Caerphilly Prospective Study 
(CaPS) and a meta-analysis," Clinical Endocrinology, vol. 70, no. 3, pp. 484-492, 2009.

[30] N. Ochs, R. Auer, D. C. Bauer et al., "Meta-analysis: subclinical thyroid dysfunction and the risk for coronary heart disease and mortality," Annals of Internal Medicine, vol. 148, no. 11, pp. 832-845, 2008.

[31] J. Karmisholt, S. Andersen, and P. Laurberg, "Variation in thyroid function tests in patients with stable untreated subclinical hypothyroidism," Thyroid, vol. 18, no. 3, pp. 303308, 2008.

[32] R. Over, S. Mannan, H. Nsouli-Maktabi, K. D. Burman, and J. Jonklaas, "Age and the thyrotropin response to hypothyroxinemia," The Journal of Clinical Endocrinology and Metabolism, vol. 95, no. 8, pp. 3675-3683, 2010.

[33] A. C. Van De Ven, R. T. Netea-Maier, M. Medici et al., "Underestimation of effect of thyroid function parameters on morbidity and mortality due to intra-individual variation," The Journal of Clinical Endocrinology and Metabolism, vol. 96, no. 12, pp. E2014-E2017, 2011.

[34] J. Karmisholt, S. Andersen, and P. Laurberg, "Interval between tests and thyroxine estimation method influence outcome of monitoring of subclinical hypothyroidism," The Journal of Clinical Endocrinology and Metabolism, vol. 93, no. 5, pp. 1634-1640, 2008.

[35] N. D. Christofides and C. P. Sheehan, "Multicenter evaluation of enhanced chemiluminescence labeled-antibody immunoassay (Amerlite-MAB(TM)) for free thyroxine," Clinical Chemistry, vol. 41, no. 1, pp. 24-31, 1995.

[36] L. De Groot, M. Abalovich, E. K. Alexander et al., "Management of thyroid dysfunction during pregnancy and postpartum: an endocrine society clinical practice guideline," Journal of Clinical Endocrinology and Metabolism, vol. 97, no. 8, pp. 2543-2565, 2012.

[37] A. Stagnaro-Green, M. Abalovich, E. Alexander et al., "Guidelines of the American Thyroid Association for the diagnosis and management of thyroid disease during pregnancy and postpartum," Thyroid, vol. 21, no. 10, pp. 1081-1125, 2011.

[38] J. E. M. Midgley and R. Hoermann, "Measurement of total rather than free thyroxine in pregnancy: the diagnostic implications," Thyroid. In press.

[39] R. Ball, D. B. Freedman, J. C. Holmes, J. E. M. Midgley, and C. P. Sheehan, "Low-normal concentrations of free thyroxin in serum in late pregnancy: physiological fact, not technical artefact," Clinical Chemistry, vol. 35, no. 9, pp. 1891-1896, 1989.

[40] E. Anckaert, K. Poppe, K. Van Uytfanghe, J. Schiettecatte, W. Foulon, and L. M. Thienpont, "FT4 immunoassays may display a pattern during pregnancy similar to the equilibrium dialysis ID-LC/tandem MS candidate reference measurement procedure in spite of susceptibility towards binding protein alterations," Clinica Chimica Acta, vol. 411, no. 17-18, pp. 1348-1353, 2010.

[41] R. Stricker, M. Echenard, R. Eberhart et al., "Evaluation of maternal thyroid function during pregnancy: the importance of using gestational age-specific reference intervals," European Journal of Endocrinology, vol. 157, no. 4, pp. 509-514, 2007.

[42] N. Kahric-Janicic, S. J. Soldin, O. P. Soldin, T. West, J. Gu, and J. Jonklaas, "Tandem mass spectrometry improves the accuracy of free thyroxine measurements during pregnancy," Thyroid, vol. 17, no. 4, pp. 303-311, 2007.

[43] J. E. M. Midgley, "Direct and indirect free thyroxine assay methods: theory and practice," Clinical Chemistry, vol. 47, no. 8, pp. 1353-1363, 2001.
[44] J. E. M. Midgley, “'All that glisters is not gold': ultrafiltration and free thyroxine measurement. With apologies to $\mathrm{W}$ Shakespeare," Clinical Biochemistry, vol. 44, no. 2-3, pp. 151153, 2011.

[45] J. W. Dietrich, M. Fischer, J. Jauch, E. Pantke, R. Gaertner, and C. R. Pickardt, "SPINA-THYR: a novel systems theoretic approach to determine the secretion capacity of the Thyroid gland," European Journal of Internal Medicine, vol. 10, article S34, 1999.

[46] J. W. Dietrich, A. Tesche, C. R. Pickardt, and U. Mitzdorf, "Thyrotropic feedback control: evidence for an additional ultrashort feedback loop from fractal analysis," Cybernetics and Systems, vol. 35, no. 4, pp. 315-331, 2004.

[47] A. Jostel, W. D. J. Ryder, and S. M. Shalet, "The use of thyroid function tests in the diagnosis of hypopituitarism: definition and evaluation of the TSH Index," Clinical Endocrinology, vol. 71, no. 4, pp. 529-534, 2009.

[48] C. A. Meier, M. N. Maisey, A. Lowry, J. Muller, and M. A. Smith, "Interindividual differences in the pituitary-thyroid axis influence the interpretation of thyroid function tests," Clinical Endocrinology, vol. 39, no. 1, pp. 101-107, 1993.

[49] B. Gereben, A. M. Zavacki, S. Ribich et al., "Cellular and molecular basis of deiodinase-regulated thyroid hormone signaling," Endocrine Reviews, vol. 29, no. 7, pp. 898-938, 2008.

[50] C. A. Spencer, J. S. LoPresti, A. Patel et al., "Applications of a new chemiluminometric thyrotropin assay to subnormal measurement," The Journal of Clinical Endocrinology and Metabolism, vol. 70, no. 2, pp. 453-460, 1990.

[51] M. K. S. Leow, "A mathematical model of pituitary-thyroid interaction to provide an insight into the nature of the thyrotropin-thyroid hormone relationship," Journal of Theoretical Biology, vol. 248, no. 2, pp. 275-287, 2007.

[52] N. Benhadi, E. Fliers, T. J. Visser, J. B. Reitsma, and W. M. Wiersinga, "Pilot study on the assessment of the setpoint of the hypothalamus- pituitary-thyroid axis in healthy volunteers," European Journal of Endocrinology, vol. 162, no. 2, pp. 323$329,2010$.

[53] H. E. Van Deventer, D. R. Mendu, A. T. Remaley, and S. J. Soldin, "Inverse log-linear relationship between thyroidstimulating hormone and free thyroxine measured by direct analog immunoassay and tandem mass spectrometry," Clinical Chemistry, vol. 57, no. 1, pp. 122-127, 2011.

[54] P. M. Clark, R. L. Holder, S. M. Haque, F. D. R. Hobbs, L. M. Roberts, and J. A. Franklyn, "The relationship between serum TSH and free $\mathrm{T}_{4}$ in older people," Journal of Clinical Pathology, vol. 65 , no. 5, pp. 463-465, 2012.

[55] K. Rothacker, N. C. Hadlow, R. Wardrop, and J. P. Walsh, "The relationship between TSH and free $\mathrm{T}_{4}$ is not log-linear and differs between genders and age groups : ASN Events," Australian Endocrine Society, pp. 1-3, 2012, http://esa-srb2012.m.asnevents.com.au.

[56] M. A. Lazar, "Thyroid hormone receptors: multiple forms, multiple possibilities," Endocrine Reviews, vol. 14, no. 2, pp. 184-193, 1993.

[57] M. F. Langlois, K. Zanger, T. Monden, J. D. Safer, A. N. Hollenberg, and F. E. Wondisford, "A unique role of the $\beta$ 2 thyroid hormone receptor isoform in negative regulation by thyroid hormone: mapping of a novel amino-terminal domain important for ligand-independent activation," The Journal of Biological Chemistry, vol. 272, no. 40, pp. 24927-24933, 1997.

[58] S. Lee, B. M. Young, W. Wan, I. H. Chan, and M. L. Privalsky, "A mechanism for pituitary-resistance to thyroid 
hormone (PRTH) syndrome: a loss in cooperative coactivator contacts by thyroid hormone receptor (TR) $\beta 2$," Molecular Endocrinology, vol. 25, no. 7, pp. 1111-1125, 2011.

[59] W. Wan, B. Farboud, and M. L. Privalsky, "Pituitary resistance to thyroid hormone syndrome is associated with $\mathrm{T}_{3}$ receptor mutants that selectively impair $\beta 2$ isoform function," Molecular Endocrinology, vol. 19, no. 6, pp. 1529-1542, 2005.

[60] M. F. Prummel, L. J. S. Brokken, G. Meduri, M. Misrahi, O. Bakker, and W. M. Wiersinga, "Expression of the thyroidstimulating hormone receptor in the folliculo-stellate cells of the human anterior pituitary," The Journal of Clinical Endocrinology and Metabolism, vol. 85, no. 11, pp. 4347-4353, 2000.

[61] A. L. Maia, I. M. Goemann, E. L. S. Meyer, and S. M. Wajner, "Type 1 iodothyronine deiodinase in human physiology and disease," Journal of Endocrinology, vol. 209, no. 3, pp. 283-297, 2011.

[62] H. J. Steinfelder, P. Hauser, Y. Nakayama et al., "Thyrotropinreleasing hormone regulation of human TSHB expression: role of a pituitary-specific transcription factor (Pit-1/GHF-1) and potential interaction with a thyroid hormone-inhibitory element," Proceedings of the National Academy of Sciences of the United States of America, vol. 88, no. 8, pp. 3130-3134, 1991.

[63] M. I. Chiamolera and F. E. Wondisford, "Minireview: thyrotropin-releasing hormone and the thyroid hormone feedback mechanism," Endocrinology, vol. 150, no. 3, pp. 1091-1096, 2009.

[64] A. Marsili, E. Sanchez, P. Singru et al., "Thyroxine-induced expression of pyroglutamyl peptidase II and inhibition of TSH release precedes suppression of TRH mRNA and requires type 2 deiodinase," Journal of Endocrinology, vol. 211, no. 1, pp. 7378, 2011.

[65] S. Grozinsky-Glasberg, A. Fraser, E. Nahshoni, A. Weizman, and L. Leibovici, "Thyroxine-triiodothyronine combination therapy versus thyroxine monotherapy for clinical hypothyroidism: meta-analysis of randomized controlled trials," The Journal of Clinical Endocrinology and Metabolism, vol. 91, no. 7, pp. 2592-2599, 2006.

[66] B. Biondi and L. Wartofsky, "Combination treatment with $\mathrm{T}_{4}$ and $\mathrm{T}_{3}$ : toward personalized replacement therapy in hypothyroidism?" The Journal of Clinical Endocrinology and Metabolism, vol. 97, no. 7, pp. 2256-2271, 2012.

[67] G. Atzmon, N. Barzilai, J. G. Hollowell, M. I. Surks, and I. Gabriely, "Extreme Longevity is associated with increased serum thyrotropin," The Journal of Clinical Endocrinology and Metabolism, vol. 94, no. 4, pp. 1251-1254, 2009.

[68] R. P. Peeters, "Thyroid function and longevity: new insights into an old dilemma," The Journal of Clinical Endocrinology and Metabolism, vol. 94, no. 12, pp. 4658-4660, 2009.

[69] P. Falaschi, A. Martocchia, A. Proietti et al., "The hypothalamic-pituitary-thyroid axis in subjects with subclinical thyroid diseases: the impact of the negative feedback mechanism," Neuroendocrinology Letters, vol. 25, no. 4, pp. 292-296, 2004.

[70] H. C. Hoftijzer, K. A. Heemstra, T. J. Visser et al., "The type 2 deiodinase ORFa-Gly3Asp polymorphism (rs12885300) influences the set point of the hypothalamus-pituitary-thyroid axis in patients treated for differentiated thyroid carcinoma," The Journal of Clinical Endocrinology and Metabolism, vol. 96, no. 9, pp. E1527-E1533, 2011.

[71] V. Panicker, C. Cluett, B. Shields et al., "A common variation in deiodinase 1 gene $D I O 1$ is associated with the relative levels of free thyroxine and triiodothyronine," The Journal of Clinical
Endocrinology and Metabolism, vol. 93, no. 8, pp. 3075-3081, 2008.

[72] B. Gencer, T.-H. Collet, V. Virgini et al., "Subclinical thyroid dysfunction and the risk of heart failure events an individual participant data analysis from 6 prospective cohorts," Circulation, vol. 126, no. 9, pp. 1040-1049, 2012.

[73] J. Jonklaas and S. J. Soldin, "Tandem mass spectrometry as a novel tool for elucidating pituitary-thyroid relationships," Thyroid, vol. 18, no. 12, pp. 1303-1311, 2008. 


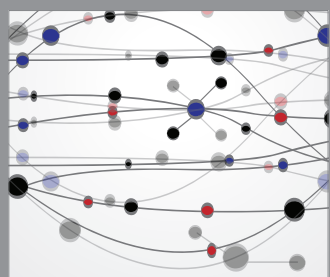

The Scientific World Journal
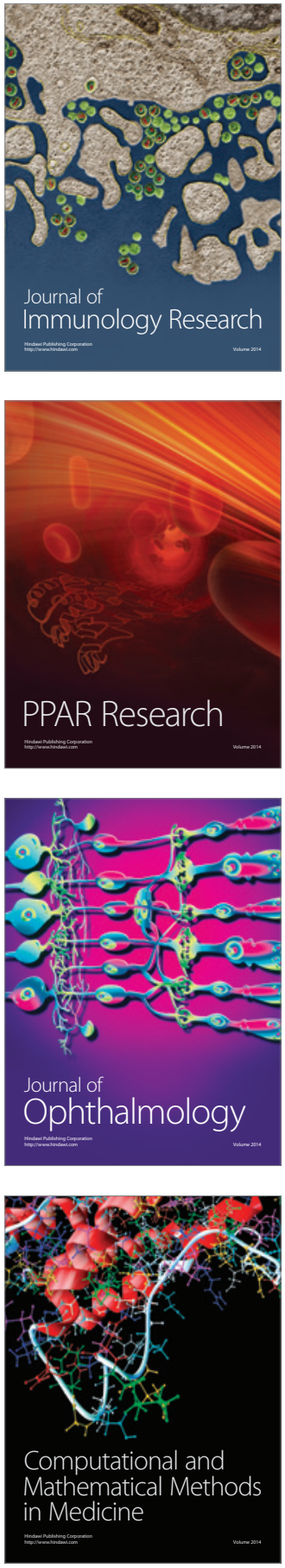

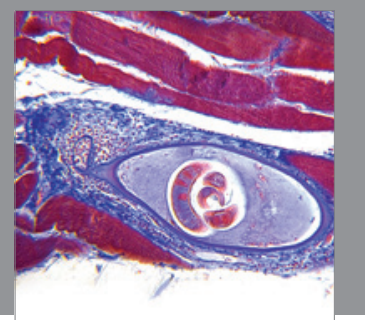

Gastroenterology

Research and Practice
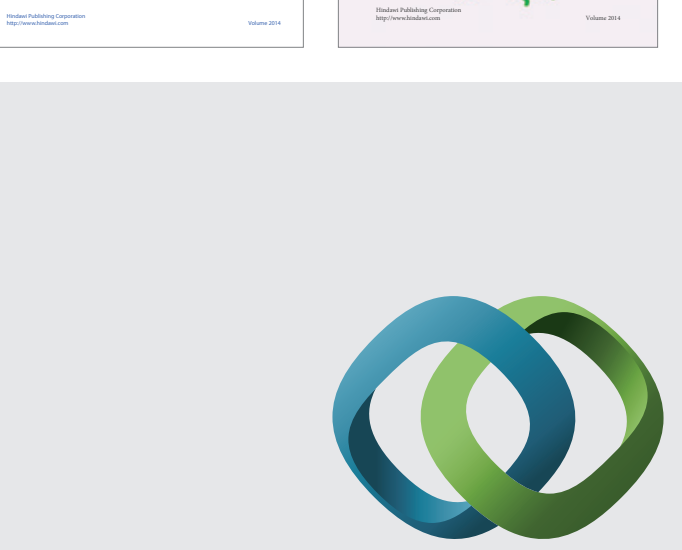

\section{Hindawi}

Submit your manuscripts at

http://www.hindawi.com
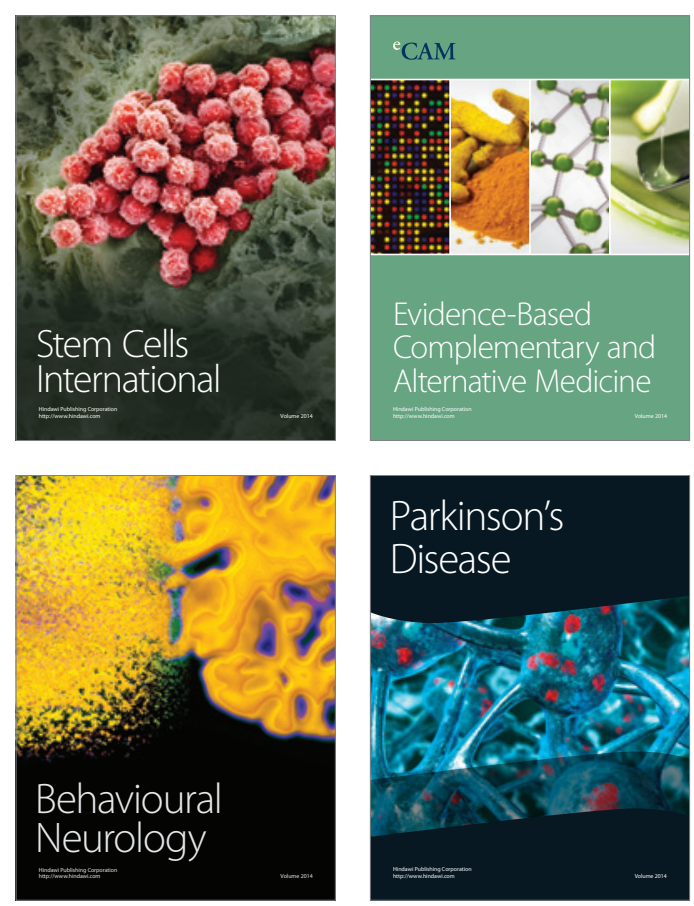

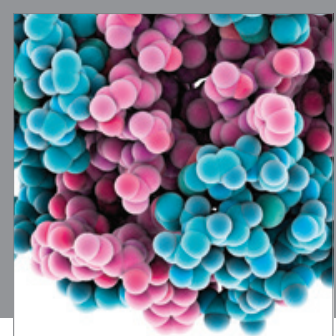

Journal of
Diabetes Research

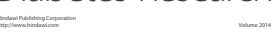

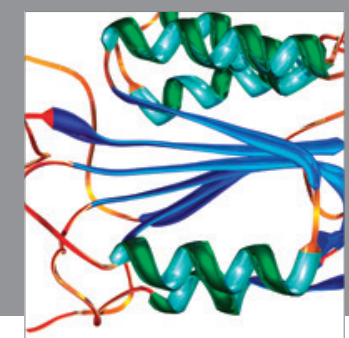

Disease Markers
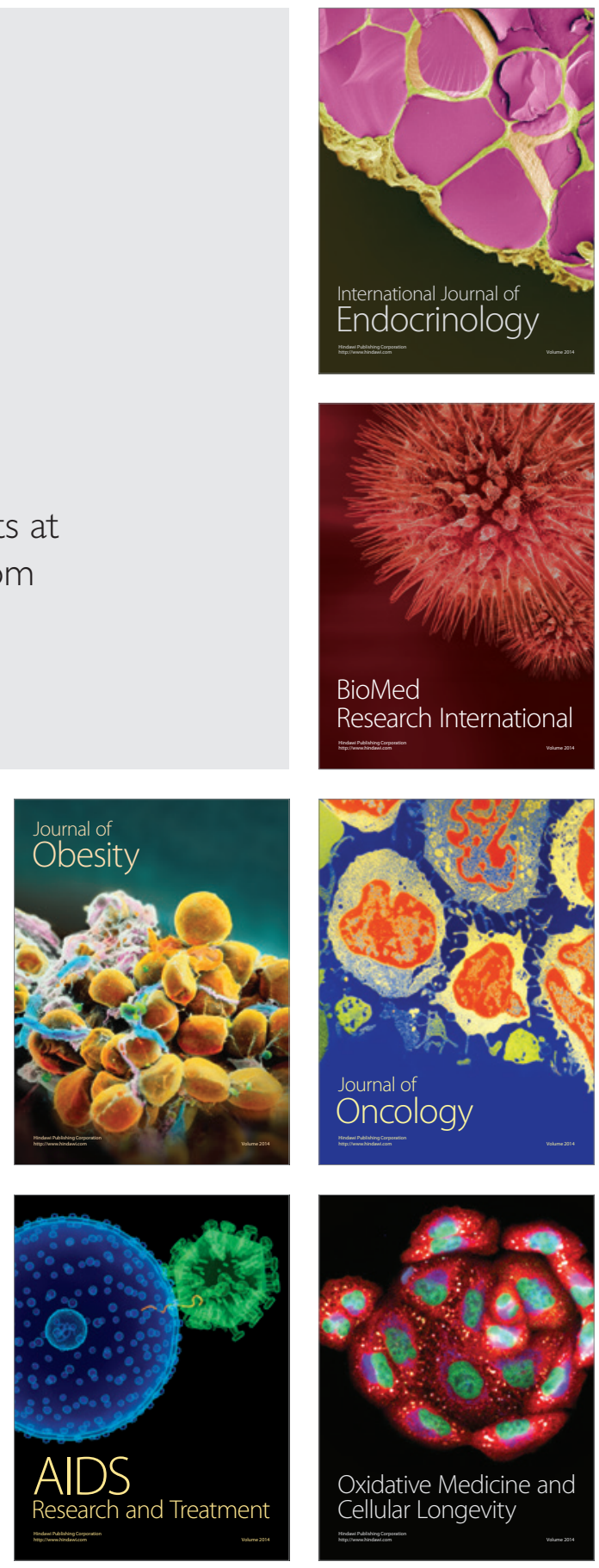